\title{
Conservation laws and invariant solutions of the nonlinear governing equations associated with a thermodynamic model of a rotating detonation engines with Korobeinikov's chemical source term
}

\author{
R. N. Ibragimov ${ }^{c}$, N. H. Ibragimov ${ }^{a, b}$, L.R. Galiakberova ${ }^{a}$ \\ ${ }^{a}$ Laboratory "Group analysis of mathematical models in natural and engineering sciences", \\ Ufa State Aviation Technical University, 450000 Ufa, Russia. \\ ${ }^{b}$ Research Centre ALGA: Advances in Lie Group Analysis, \\ Department of Mathematics and Natural Sciences, \\ Blekinge Institute of Technology, SE-371 79 Karlskrona, Sweden. \\ ${ }^{c}$ GE Global Research \\ 1 Research Circle \\ Niskayuna, NY 12309, USA
}

July 4, 2015

\begin{abstract}
The nonlinear governing gas dynamics equations that are used as a descriptor of a rotating detonation engine are investigated from the group theoretical standpoint. The equations incorporate approximation of the Korobeinkov's chemical reaction model that are used to describe the two-dimensional detonation field on a surface of a two-dimensional cylindrical chamber without thickness. The transformations that leave the equations invariant are found. On the basis of these transformations, the conservation equations were constructed and the invariant solutions were obtained for specific form of the equation of state, for which the equations are nonlinearly self-adjoint. The invariant solutions are given in terms of the functions that satisfy nonlinear ordinary differential equations. The above reduction simplifies the analysis of the original nonlinear system of partial differential equations on a surface of rotating cylinder.
\end{abstract}

\section{Introduction}

Within the last 15 years, there has been a considerable amount of research into developing engines utilizing detonation waves for air-breathing propulsion, to the point where propulsion engines are being developed and tested ([18]; [19]).

Rotating Detonation Engine (RDE) takes a different approach toward realizing the efficiency the detonation cycle ([2]; [21]). By allowing the detonation to propagate azimuthally around an annular combustion chamber, the kinetic energy of the inflow can be held to a relatively low value, and thus RDE can use most of the compression for gains in efficiency, while the flow field matches the steady detonation cycler closely $([15])$. 


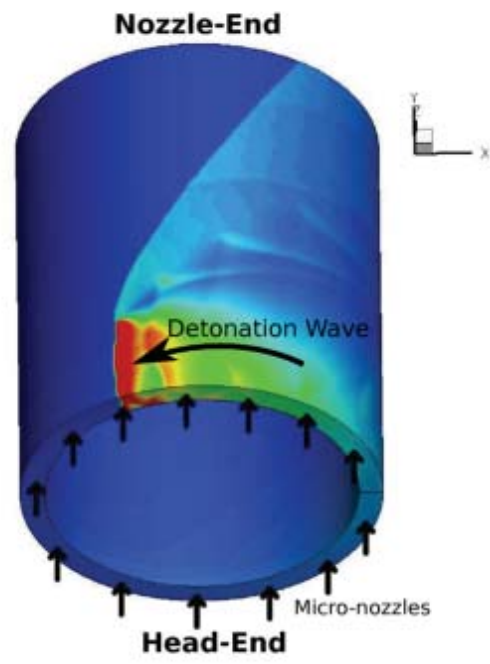

Figure 1: Example schematic of a Rotating Detonation Engine. The combustion chamber is an annular ring, where the mean direction of a flow is from the head end (bottom in figure) to the exit (top). Source: Kailasanath

A basic mechanism of RDE is shown in Fig. 1. The micro-nozzles flow in a premixture of fuel and air or oxygen, and a detonation propagates circumferentially around the combustion chamber consuming the freshly injected mixture. The gas then expands azimuthally and axially, and is either subsonic or supersonic (or both), depending on the back pressure at the outlet plane. Similarly to our previous studies in [5] and [9], where the dynamics of small perturbations of a certain class of stationary exact solutions to the nonlinear governing equations of geophysical stratified fluid motion in a cylindrical wave field was investigated, the flow in RDE has a very strong circumferential aspect [23]. However, unlike in [5], where a such flow was in virtue of stratification and the effects of rotation due to Coriolis force, for RDE, a such circumferential aspect is due to detonation wave propagation ([25]). Similar to our approach in [5], since for RDE, the radial dimension is typically small compared to the azimuthal and axial dimension, we shall neglect the radial dependence in our modeling, which allows the RDE to be unrolled into two dimensional flow. Another similar approach for describing the dynamics of weakly nonlinear waves for compressible inviscous flows rotating in an annular ring has been developed in [9] and [8] for stratified and homogeneous fluids (see [7]; [6]; [10]).

In this paper, we analyze the nonlinear governing gas dynamics equations that are used as a descriptor of a rotating detonation engine from the group theoretical standpoint. The equations incorporate the simplified version of the Korobeinikov et al. [17] chemical reaction model that is used to describe the two-dimensional detonation field on a surface of a twodimensional cylindrical chamber without thickness. First, we find the one-parameter group of transformations that leave the equations in question to be invariant with respect to these transformations. Next, on the basis of these transformations, the conservation equations will be constructed and the invariant solutions will be obtained for specific form of the equation of state, for which the equations are nonlinearly self-adjoint. The invariant solutions are then obtained in terms of the functions that satisfy nonlinear ordinary differential equations. The 
above reduction simplifies the analysis of the original nonlinear system of partial differential equations on a surface of rotating cylinder.

\section{Model}

From the standpoint of a mathematical modeling, the gas motion in the domain $\Omega \subset R^{4}(x, t)$ is the set of functions $\vec{u}, \rho, p, \epsilon$, defined in $\Omega$ and satisfying the standard gas dynamic equations with an additional chemical source term $\dot{\omega}$ (see e.g.[20];[4]),

$$
\begin{aligned}
D \rho+\rho \operatorname{div}(\vec{u}) & =\dot{\omega}, \\
D \vec{u}+\frac{1}{\rho} \nabla p & =0, \\
D \epsilon+\frac{p}{\rho} \operatorname{div}(\vec{u}) & =0,
\end{aligned}
$$

where $\vec{u}=(u, v, w)$ is a velocity vector, $\rho$ is a density, $p$ is a pressure, $\epsilon$ is a specific internal energy,

$$
D=\frac{\partial}{\partial t}+\vec{u} \cdot \nabla=\frac{\partial}{\partial t}+u \frac{\partial}{\partial x}+v \frac{\partial}{\partial y}+w \frac{\partial}{\partial z}
$$

is the differential operator and $\dot{\omega}$ is the rate of change of a mixture density due to chemical reaction. We also not that the system (1) - (3) is being closed by the equation of state

$$
p=f(\rho, S) .
$$

Due to Eq. (1), we can rewrite (3) as

$$
D \epsilon+p D V+\frac{p \dot{\omega}}{\rho^{2}}=0
$$

where we denote $V=1 / \rho$, so that we associate $V$ with the specific gas volume (the volume of a unit mass). Thus, the Eq. (6) represents the balance between the increase in the inertial energy plus the work of expansion of the portion of the gas and the rate of change of the density in gas due to chemical reaction. Next, let us denote the absolute temperature by $T$. Due to Eq. (5), the first law of thermodynamics yields

$$
D S=-\frac{p \dot{\omega}}{T \rho^{2}}
$$

where $T$ is the absolute temperature.

For further analysis, it is convenient to eliminate the entropy by using the equation: $D p=$ $f_{\rho} D \rho+f_{s} D s$, where the low indexes represent partial derivatives. As follows from the definition of the speed of sound $c$ (see e.g. [20]),

$$
c^{2}=\left.\frac{d p}{d \rho}\right|_{S=\text { const }}=f_{\rho}(\rho, S)
$$

one can see that the equation (7) is equivalent to the relation

$$
D p=c^{2} D \rho-\frac{f_{S} p \dot{\omega}}{T \rho^{2}} .
$$


Thus we can rewrite the original model (1) - (3) as

$$
\begin{aligned}
D \vec{u}+\frac{1}{\rho} \nabla p & =0, \\
b D p+\operatorname{div}(\vec{u}) & =\left(1-\frac{f_{S} p}{T c^{2} \rho^{2}}\right) \frac{\dot{\omega}}{\rho}, \\
D S & =-\frac{p}{T \rho} \frac{\dot{\omega}}{\rho},
\end{aligned}
$$

in which the values $\rho$ and $b=1 / \rho c^{2}$ are considered as functions of $p$ and $S$. We also remark that, the entropy $S$ and the energy $\epsilon=\Xi(V, T)$ are related by the relation (see also [20] )

$$
S=R \ln V+\int \Xi^{\prime}(T) d \ln T
$$

i.e. in reality, the energy depends on the temperature and the solution $S$ of the model (10) (12) yields also the solution for the energy.

We next use the analogue of the Korobeinikov et al. [17] chemical reaction to approximate the chemical source term $\dot{\omega}$ by the relation (see also [3])

$$
\dot{\omega} \approx-\alpha p^{n_{1}} \rho^{l_{1}} \exp \left(-\frac{E^{*}}{R T}\right)
$$

in which $R$ is a gas constant, and the parameters $\alpha, m_{1}, n_{1}, l_{1}$ are $E^{*}$ (representing the energy activation) are determined from the experimental data ([1]; [2]). Without loss of generality we can normalize the set of the data by taking $n_{1}=1$ and for the sake of simplicity of calculations, we shall set $l_{1}=1$. Usually (see e.g. [17]; [24]), the relation (13) is used to model equation an accumulation of active radicals in a particle during a chemical reaction. At the initial time, $\omega=0$, and the induction period stops at $\omega=1$. After this, the second stage of the reaction begins, which is described by the equation, similar to the relation (13). However, we limit ourselves by a simplified version of the chemical reaction and don't include the higher stages of reactions.

The original model (10) - (12) consists of four nonlinear equations for the unknowns $(u, v, p, S)$, in which $\vec{u}=(u, v)$ is the velocity vector, $p$ is a pressure, $\rho$ is density, $S$ is the entropy, $r=$ const is a fixed radius of the cylinder, along which we study the gas propagation, and $b=1 / \rho^{2} c$ (in which we can consider the sound speed $c$ either to be a constant, or, for example as a function of $(p, S)$ via relation $\left.c^{2}=d p / d \rho\right)$.

The calculations are based on several assumptions and approximations. The basic ones are:

$$
c=c_{0}
$$

and the equation of state (5) is simplified to the form.

$$
p=f(\rho) .
$$

Another simplifying assumption in this paper is based on considering a special case when the absolute temperature $T$ is sufficiently high, which corresponds to the ignition process in a turbine (e.g. in the moment when an airplane takes off, the absolute temperature is much 


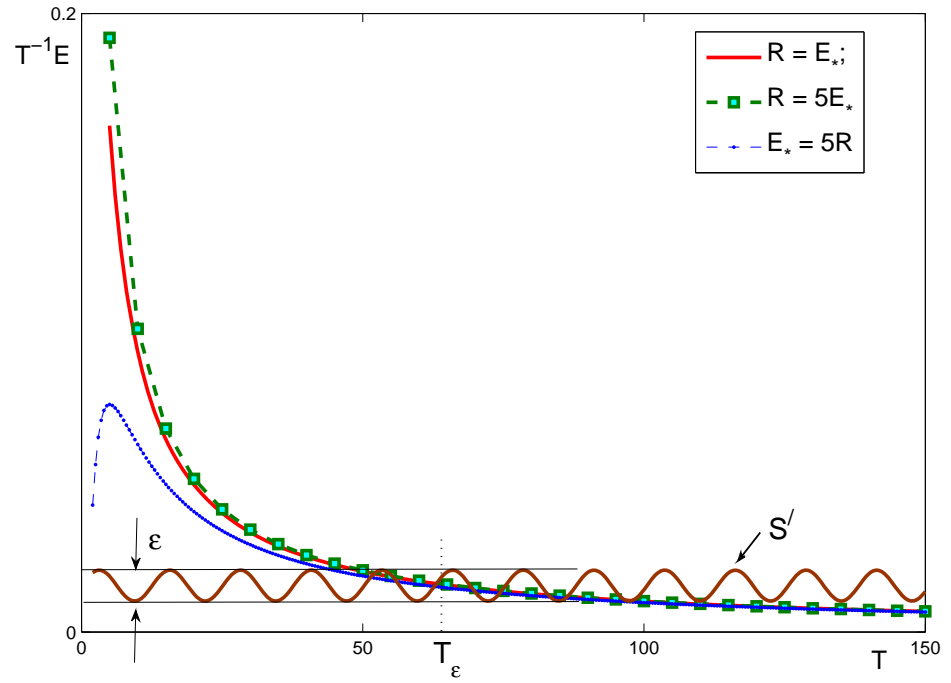

Figure 2: Qualitative behavior of $F(T)$ at different values of $E^{*}$ and $R$.

larger than the numerical values of the pressure and density). In this case, assuming $\alpha>0$ in (14), the term

$$
F=\frac{1}{T} \exp \left(-\frac{E^{*}}{R T}\right)
$$

appearing in the right side of Eq. (12) can be neglected at certain order of accuracy, by considering the values of $T$ greater than some given value $T_{\varepsilon}$, for which $F$ is less than some given small value $\varepsilon$, as shown schematically in Figure 2, which is used to visualize a qualitative behavior of $F$ at different values of parameters $E^{*}$ and $R$.

As been discussed in [22], when modeling equilibrium rocket motor performance calculations, usually, viscous forces are taken to be small compared to pressure forces and the heat transferred through the walls is considered to be small compared to the stagnation enthalpy of the gases in the chamber; thus, it is usually assumed that the camber walls are frictionless and adiabatic. As a result, as discussed in [1], the gas flow is isentropic. Based on this observation, we will simplify the model by assuming that

$$
S=S_{0}+\varepsilon S^{\prime}
$$

where $S_{0}=$ const, $\varepsilon$ is a given sufficiently small number (which depends on the experimental data for $E^{*}$ and $R$ ) and $S^{\prime}$ is a bounded function of the absolute temperature $T$ as demonstrated in Figure 2. In particular, the recent experimental studies on rotating detonation of rocket performance reported in [16] confirm the validity of the assumption of homogeneous mixing and adiabatic (or almost adiabatic) combustion, i.e. a heat transfer outside of the system is negligible, which corresponds to the case, when $S^{\prime} \approx$ const. in (18).

Combined with the assumption (16), up to certain order of accuracy, the last equation (12) in the model can be neglected by dropping the right side and assuming $S=S_{0}$ at the leading order. 
Next, in order to describe the gas-filed in an annular chamber without thickness, as shown in Fig. 1, we introduce cylindrical coordinates $x=r \cos \theta, y=r \sin \theta, z=z$ so that the rate-of-strain tensor is

$$
e_{r r}=\frac{\partial u_{r}}{\partial r}, \quad e_{\theta \theta}=\frac{1}{r} \frac{\partial u_{\theta}}{\partial \theta}, \quad e_{r \theta}=\frac{r}{2} \frac{\partial}{\partial r}\left(\frac{u_{\theta}}{r}\right)+\frac{1}{2 r} \frac{\partial u_{r}}{\partial \theta} .
$$

Within the approximation (15), (16) and (18), the resulting two-dimensional version of the model is simplified to the form:

$$
\begin{aligned}
& F_{1}=\rho\left(\frac{\partial u}{\partial t}+\frac{u}{r} \frac{\partial u}{\partial \theta}+v \frac{\partial u}{\partial z}\right)+\frac{1}{r} \frac{\partial}{\partial \theta} f(\rho)=0, \\
& F_{2}=\rho\left(\frac{\partial v}{\partial t}+\frac{u}{r} \frac{\partial v}{\partial \theta}+v \frac{\partial v}{\partial z}\right)+\frac{\partial}{\partial z} f(\rho)=0, \\
& F_{3}=\frac{1}{\rho^{2} c_{0}}\left(\frac{\partial f(\rho)}{\partial t}+\frac{u}{r} \frac{\partial f(\rho)}{\partial \theta}+v \frac{\partial f(\rho)}{\partial z}\right)+\frac{1}{r} \frac{\partial u}{\partial \theta}+\frac{\partial v}{\partial z}+\alpha E f(\rho)=0,
\end{aligned}
$$

where $u=u_{\theta}, v=u_{z}$ and we denote $E=e^{-\frac{E^{*}}{R T}}$. The model $(20)$ - (21) represents a nonlinear system of three equations for three unknowns $(u, v, \rho)$.

\section{$3 \quad$ Preliminaries on symmetry groups and invariant solutions}

In terms of group theoretical modeling, the dynamics of the system in the question can often be described by means of the formal Lagrangian and the adjoint equations. The detailed review of this subject can be found e.g. in [11] and in [4] and here we emphasize that part of the review in the latter references that is essential in the frame of the current work.

Let $x=\left(x^{1}, \ldots, x^{n}\right)$ be $n$ independent variables, and $u=\left(u^{1}, \ldots, u^{m}\right)$ be $m$ dependent variables. We will deal with one-parameter group $G$ of transformations of the form

$$
\begin{aligned}
\bar{x}^{i} & =\varphi^{i}(x, a),\left.\quad \varphi^{i}\right|_{a=0}=x^{i}, \\
\bar{u}^{\alpha} & =\psi^{\alpha}(x, u, a),\left.\quad \psi^{\alpha}\right|_{a=0}=u^{\alpha},
\end{aligned}
$$

depending on a continuous parameter $a$. The infinitesimal transformation of the group $G$ are written

$$
\bar{x}^{i} \approx x^{i}+a \xi^{i}(x), \quad \bar{u}^{\alpha} \approx u^{\alpha}+a \eta^{\alpha}(x, u) .
$$

The generator of the group $G$ is the linear first-degree differential operator

$$
X=\xi^{i}(x) \frac{\partial}{\partial x^{i}}+\eta^{\alpha}(x, u) \frac{\partial}{\partial u^{\alpha}},
$$

where we employ the usual convention on summation over repeated indices.

Let us consider a (linear or nonlinear) system of partial differential equations

$$
F_{\alpha}\left(x, u, u_{(1)}, \ldots, u_{(s)}\right)=0, \quad \alpha=1, \ldots, m,
$$


where $u_{(1)}=\left\{u_{i}^{\alpha}\right\}, u_{(2)}=\left\{u_{i j}^{\alpha}\right\}, \ldots$ denote the sets of partial derivatives of the respective orders, e.g.

$$
u_{i}^{\alpha}=D_{i}\left(u^{\alpha}\right), \quad u_{i j}^{\alpha}=D_{i}\left(u_{j}^{\alpha}\right)=D_{i} D_{j}\left(u^{\alpha}\right), \ldots
$$

where $D_{i}$ is the operator of total differentiation with respect to $x^{i}$ :

$$
D_{i}=\frac{\partial}{\partial x^{i}}+u_{i}^{\alpha} \frac{\partial}{\partial u^{\alpha}}+u_{i j}^{\alpha} \frac{\partial}{\partial u_{j}^{\alpha}}+\ldots, \quad i=1, \ldots, n .
$$

Even though the operators $D_{i}$ are given by formal infinite sums, their action $D_{i}(f)$ is well defined for functions $f\left(x, u, u_{(1)}, \ldots\right)$ depending on a finite number of the variables $x, u, u_{(1)}, u_{(2)}, \ldots$ The usual summation convention on repeated indices $\alpha$ and $i$ is assumed in expressions like Eq. (20).

For example, in terms of the operators $D_{i}$, the derivatives $u_{(1)}$ and $u_{(2)}$ can also be written as

$$
u_{(1)}=u_{i}^{\alpha}=\frac{\partial u^{\alpha}(x)}{\partial x^{i}}, \quad u_{(2)}=u_{i j}^{\alpha}=\frac{\partial^{2} u^{\alpha}(x)}{\partial x^{i} \partial x^{j}} .
$$

The group $G$ is called a symmetry group (or admitted group) for Eqs. (27), if the system (27) has the same form when it is written in the new variables $\bar{x}, \bar{u}$ :

$$
F_{\alpha}\left(\bar{x}, \bar{u}, \bar{u}_{(1)}, \ldots, \bar{u}_{(s)}\right)=0, \quad \alpha=1, \ldots, m,
$$

The effective way for constructing the symmetry group for Eqs. (27) is to solve the determining equations

$$
\left.X_{(s)} F_{\alpha}\left(x, u, u_{(1)}, \ldots, u_{(s)}\right)^{i}\right|_{(27)}=0
$$

for the generator (26) of the symmetry group $G$, where $X_{(s)}$ is the $s^{\text {th }}$ prolongation for the generator $X$ of the group $G$, and the notation $\left.\right|_{(27)}$ means evaluated on Eqs. (27) (see [4] for the nomenclature).

The admitted group $G$ maps any solution of the system (27) into a solution of the same system. A solution that mapped by $G$ into itself is knows as an invariant solution with respect to the group $G$. The invariant solutions for a group with a generator $X$ are obtained by finding the invariants $J(x, u)$ of the group $G$. The invariants are found by solving the first-order linear partial differential equation

$$
X(J) \equiv \xi^{i}(x) \frac{\partial J}{\partial x^{i}}+\eta^{\alpha}(x, u) \frac{\partial J}{\partial u^{\alpha}}=0,
$$

or its characteristic system

$$
\frac{d x^{1}}{\xi^{1}(x)}=\ldots=\frac{d x^{n}}{\xi^{n}(x)}=\frac{d u^{1}}{\eta^{1}(x, u)}=\frac{d u^{m}}{\eta^{m}(x, u)} .
$$

It is manifested from Eqs. (33) that the group has $n+m-1$ functionally independent invariants of the following form:

$$
\lambda_{1}(x), \ldots, \lambda_{n-1}(x), \quad \Phi_{1}(x), \ldots, \Phi_{m}(x, u) .
$$


Then we let

$$
\Phi_{\alpha}=\phi_{\alpha}\left(\lambda_{1}, \ldots, \lambda_{n-1}\right),
$$

solve these equations for $u^{1}, \ldots, u^{m}$, substitute the resulting expressions for $u$ in Eqs. (27) and obtain a system of partial differential equations for the unknown functions $\phi_{\alpha}\left(\lambda_{1}, \ldots, \lambda_{n-1}\right)$ depending on $n-1$ variables.

If we require the invariance with respect to a symmetry group with $n-1$ linearly independent generators, then we will have only invariant of the form $\lambda(x)$, and the system (27) will be reduced to ordinary differential equations for unknown functions $\phi_{\alpha}(\lambda)$.

\subsection{Nonlinear self-adjointness}

We use the definition of the nonlinear self-adjointness of systems of nonlinear partial differential equations [14]. According to this definition the adjoint system to the system (31) has the form

$$
F_{\alpha}^{*}\left(x, u, w, u_{(1)}, w_{(1)}, \ldots, u_{(s)}, w_{(s)}\right)=0, \quad \alpha=1, \ldots, m .
$$

Here the adjoint operator $F_{\alpha}^{*}$ is defined by

$$
F_{\alpha}^{*}\left(x, u, w, u_{(1)}, w_{(1)}, \ldots, u_{(s)}, w_{(s)}\right)=\frac{\delta \mathcal{L}}{\delta u^{\alpha}},
$$

where

$$
\begin{aligned}
\frac{\delta}{\delta u^{\alpha}} & =\frac{\partial}{\partial u^{\alpha}}-D_{i} \frac{\partial}{\partial u_{i}^{\alpha}}+D_{i} D_{j} \frac{\partial}{\partial u_{i j}^{\alpha}}-D_{i} D_{j} D_{k} \frac{\partial}{\partial u_{i j k}^{\alpha}}+\ldots \\
& =\frac{\partial}{\partial u^{\alpha}}+\sum_{s=1}^{\infty}(-1)^{s} D_{i_{1}} \cdots D_{i_{s}} \frac{\partial}{\partial u_{i_{1} \cdots i_{s}}^{\alpha}}, \quad \alpha=1, \ldots, m
\end{aligned}
$$

is the variational derivative (the Euler-Lagrange operator).

In Equation $(37) w=\left(w^{1}, \ldots, w^{m}\right)$ are new dependent variables, $\mathcal{L}$ is the formal Lagrangian for the system (31) given by

$$
\mathcal{L}=\sum_{\alpha=1}^{m} w^{\alpha} F_{\alpha}\left(x, u, u_{(1)}, \ldots, u_{(s)}\right) .
$$

The system (31) is said to be nonlinearly self-adjoint if there exist a substitution

$$
w^{\alpha}=\varphi_{\alpha}(x, u), \quad \alpha=1, \ldots, m,
$$

where not all $\varphi_{\alpha}(x, u)$ vanish identically, such that the adjoint system (36) is satisfied for all solutions of the system (31) after eliminating the variables $\mu^{\alpha}$ by the substitution (39). This definition is equivalent to the requirement that the equations

$$
F_{\alpha}^{*}\left(x, u, \varphi, u_{(1)}, \varphi_{(1)}, \ldots, u_{(s)}, \varphi_{(s)}\right)=\mu_{\alpha}^{\beta}(x, u) F_{\beta}\left(x, u, u_{(1)}, \ldots, u_{(s)}\right)
$$

hold identically in $x, u, u_{(1)}, \ldots, u_{(s)}$ for all $\alpha=1, \ldots, m$. Here $\mu_{\alpha}^{\beta}(x, u)$ are indeterminate coefficients, $\varphi$ is the $m$-dimensional vector

$$
\varphi=\left(\varphi_{1}(x, u), \ldots, \varphi_{m}(x, u)\right)
$$

and $\varphi_{(1)}, \ldots, \varphi_{(s)}$ denote its derivatives. 


\subsection{Conservation laws for nonlinearly self-adjoint equations}

The general formula for constructing conservation laws

$$
D_{i}\left(C^{i}\right)=0
$$

for nonlinearly self-adjoint equations (31) with known infinitesimal symmetries

$$
X=\xi^{i} \frac{\partial}{\partial x^{i}}+\eta^{\alpha} \frac{\partial}{\partial u^{\alpha}}
$$

is given in [14]. In the case of first-order systems (31) this formula has the following simple form:

$$
C^{i}=\left(\eta^{\alpha}-\xi^{j} u_{j}^{\alpha}\right) \frac{\partial \mathcal{L}}{\partial u_{i}^{\alpha}},
$$

where the additional variables $w^{1}, \ldots, w^{m}$ involved in the formal Lagrangian $\mathcal{L}$ should be eliminated by using the substitution (39).

This approach has been applied in our previous work in [13] to the system of nonlinear equations describing wave beams propagation in the ocean.

\section{Group classification and symmetries}

In case when $p=f(\rho)$ is an arbitrary function, symmetries of the system (20) - (22) are the following:

$$
\begin{gathered}
X_{1}=\frac{\partial}{\partial t}, \quad X_{2}=\frac{\partial}{\partial \theta}, \quad X_{3}=\frac{\partial}{\partial z}, \\
X_{4}=t \frac{\partial}{\partial \theta}+r \frac{\partial}{\partial u}, \quad X_{5}=t \frac{\partial}{\partial z}+\frac{\partial}{\partial v}, \\
X_{6}=z \frac{\partial}{\partial \theta}-r^{2} \theta \frac{\partial}{\partial z}+r v \frac{\partial}{\partial u}-r u \frac{\partial}{\partial v} .
\end{gathered}
$$

Additional symmetry $X_{7}$ exist only in case when $p=a \rho^{2}(a \neq 0)$ :

$$
X_{7}=4 t \frac{\partial}{\partial t}+3 \theta \frac{\partial}{\partial \theta}+3 z \frac{\partial}{\partial z}-u \frac{\partial}{\partial u}-v \frac{\partial}{\partial v}-2 \rho \frac{\partial}{\partial \rho} .
$$

Without loss of generality, we can set $a=c_{0}$.

\subsection{Nonlinear self-adjointness of the model}

Adjoint system to (20) -( 22):

$$
F_{1}^{*}=0, \quad F_{2}^{*}=0, \quad F_{3}^{*}=0,
$$

where

$$
F_{1}^{*}=\frac{\delta \mathcal{L}}{\delta u}, \quad F_{2}^{*}=\frac{\delta \mathcal{L}}{\delta v}, \quad F_{3}^{*}=\frac{\delta \mathcal{L}}{\delta \rho}
$$

with

$$
\mathcal{L}=w^{1} F_{1}+w^{2} F_{2}+w^{3} F_{3} .
$$


Here $w^{1}, w^{2}, w^{3}$ are new dependent variables.

The calculation gives

$$
\begin{aligned}
F_{1}^{*}= & \frac{f^{\prime}(\rho)}{c_{0}^{2} \rho^{2}} \frac{\partial \rho}{\partial \theta} w^{3}-\left(\frac{\partial \rho}{\partial t}+\frac{u}{r} \frac{\partial \rho}{\partial \theta}+v \frac{\partial \rho}{\partial z}\right) w^{1}+ \\
& \left(\frac{w^{2}}{r} \frac{\partial v}{\partial \theta}-\frac{\partial w^{1}}{\partial t}-\frac{u}{r} \frac{\partial w^{1}}{\partial \theta}-v \frac{\partial w^{1}}{\partial z}\right)-\frac{1}{r} \frac{\partial w^{3}}{\partial \theta} \\
F_{2}^{*}= & \frac{f^{\prime}(\rho)}{c_{0}^{2} \rho^{2}} \frac{\partial \rho}{\partial z} w^{3}-\left(\frac{\partial \rho}{\partial t}+\frac{1}{r}\left[\rho \frac{\partial u}{\partial \theta}+u \frac{\partial \rho}{\partial \theta}\right]+v \frac{\partial \rho}{\partial z}\right) w^{2}+ \\
& \rho\left(w^{1} \frac{\partial u}{\partial z}-\frac{\partial w^{2}}{\partial t}-\frac{u}{r} \frac{\partial w^{2}}{\partial \theta}-v \frac{\partial w^{2}}{\partial z}\right)-\frac{\partial w^{3}}{\partial z}, \\
F_{3}^{*}= & \left(\frac{\partial u}{\partial t}+\frac{u}{r} \frac{\partial u}{\partial \theta}+v \frac{\partial u}{\partial z}\right) w^{1}+\left(\frac{\partial v}{\partial t}+\frac{u}{r} \frac{\partial v}{\partial \theta}+v \frac{\partial v}{\partial z}\right) w^{2}+ \\
& \left(\alpha E w^{3}-\frac{1}{r} \frac{\partial w^{1}}{\partial \theta}-\frac{\partial w^{2}}{\partial z}-\frac{\mu}{c_{0} \rho^{2}}\right) f^{\prime}(\rho),
\end{aligned}
$$

where

$$
\mu=\frac{\partial w^{3}}{\partial t}+\frac{1}{r}\left[u \frac{\partial w^{3}}{\partial \theta}+w^{3} \frac{\partial u}{\partial \theta}\right]+v \frac{\partial w^{3}}{\partial z}+w^{3} \frac{\partial v}{\partial z}
$$

System (20) -(22) will be nonlinearly self-adjoint if there exists a substitution

$$
w^{1}=\varphi_{1}(t, \theta, z, u, v, \rho), \quad w^{2}=\varphi_{2}(t, \theta, z, u, v, \rho), \quad w^{3}=\varphi_{2}(t, \theta, z, u, v, \rho)
$$

such that the following equations are satisfied:

$$
\left.F_{1}^{*}\right|_{(54)}=\mu_{1}^{i} F_{i},\left.\quad F_{2}^{*}\right|_{(54)}=\mu_{2}^{i} F_{i},\left.\quad F_{3}^{*}\right|_{(54)}=\mu_{3}^{i} F_{i}
$$

We obtain that the original model (20) -( 22) is nonlinear self-adjoint only in case of certain $f(\rho)$, namely when

$$
f(\rho)=-c_{0} \rho^{2}+c_{1}, c_{1}=\text { const } .
$$

Substitution, that provides nonlinear self-adjointness, takes the form

$$
w^{1}=0, \quad w^{2}=0, \quad w^{3}=\frac{1}{\rho^{2}} \mathrm{e}^{\alpha E c_{1} t} .
$$

The nonlinear self-adjointness condition is satisfied in the following form:

$$
\left.F_{1}^{*}\right|_{(57)}=0,\left.\quad F_{2}^{*}\right|_{(57)}=0,\left.\quad F_{3}^{*}\right|_{(57)}=\frac{2 r}{\rho^{3}} \mathrm{e}^{\alpha E c_{1} t} F_{3}
$$

In the case $c_{1} \neq 0$ application of the equation (42) to the time translation symmetry $X_{1}$ from (43) gives the conserved vector

$$
C^{t}=\left(\frac{1}{\rho^{2}}-\frac{c_{0}}{c_{1}}\right) \mathrm{e}^{\alpha E c_{1} t}, \quad C^{\theta}=\frac{u}{r \rho^{2}} \mathrm{e}^{\alpha E c_{1} t}, \quad C^{z}=\frac{v}{\rho^{2}} \mathrm{e}^{\alpha E c_{1} t} .
$$


When $c_{1}=0$, application of the equation (42) to the additional symmetry $X_{7}$ given by (46) leads to the following conserved vector:

$$
C^{t}=\frac{1}{\rho^{2}}-\alpha E c_{0} t, \quad C^{\theta}=\frac{u}{r \rho^{2}}, \quad C^{z}=\frac{v}{\rho^{2}} .
$$

The remaining symmetries give only trivial conservation laws. Moreover, the calculation made by using the definition (41) of conservation laws shows that (59) and (60) are the only conserved vectors depending on $t, \theta, z, u, v, \rho$.

\section{Invariant solutions}

Without loss of generality, we can set $c_{1}=0$ in (56). Then the model (20) - (22) with

$$
f(\rho)=-c_{0} \rho^{2}
$$

is written as

$$
\begin{gathered}
r \frac{\partial u}{\partial t}+u \frac{\partial u}{\partial \theta}+r u \frac{\partial u}{\partial z}-2 c_{0} \frac{\partial \rho}{\partial \theta}=0 \\
r \frac{\partial v}{\partial t}+u \frac{\partial v}{\partial \theta}+r v \frac{\partial v}{\partial z}-2 c_{0} r \frac{\partial \rho}{\partial z}=0 \\
2\left(r \frac{\partial \rho}{\partial t}+u \frac{\partial \rho}{\partial \theta}+r v \frac{\partial \rho}{\partial z}\right)+\left(\rho \frac{\partial u}{\partial \theta}+r \rho \frac{\partial v}{\partial z}-\alpha r E c_{0} \rho^{3}\right)=0
\end{gathered}
$$

where $E \neq 0, \alpha \neq 0, c_{0} \neq 0$ and $r \neq 0$.

\subsection{Invariant solution with respect to $X_{6}, X_{7}$}

Invariants of $X_{6}, X_{7}$ :

$$
\begin{gathered}
\lambda=t^{-3 / 2}\left(r^{2} \theta^{2}+z^{2}\right), \\
J_{1}=t^{1 / 2} \rho, \quad J_{2}=t^{1 / 2}\left(u^{2}+v^{2}\right), \quad J_{3}=t^{-1 / 2}(r \theta v-z u) .
\end{gathered}
$$

Invariant solution has the form

$$
J_{1}=F(\lambda), \quad J_{2}=G(\lambda), \quad J_{3}=H(\lambda) .
$$

Hence

$$
\begin{aligned}
\rho & =t^{-\frac{1}{2}} F(\lambda), \\
u^{2}+v^{2} & =t^{-\frac{1}{2}} G(\lambda), \\
r \theta v-z u & =t^{\frac{1}{2}} H(\lambda) .
\end{aligned}
$$

For any $G(\lambda)$ from (68) - (70) it is possible to find function $Q(\lambda)$ such that

$$
G(\lambda)=\frac{1}{\lambda}\left(Q^{2}(\lambda)+H^{2}(\lambda)\right) .
$$


Then explicit expressions can be written for $u$ and $v$ from (52):

$$
\begin{aligned}
& u=\frac{t^{1 / 2}}{r^{2} \theta^{2}+z^{2}}(-z H(\lambda) \pm r \theta Q(\lambda)), \\
& v=\frac{t^{1 / 2}}{r^{2} \theta^{2}+z^{2}}(r \theta H(\lambda) \pm z Q(\lambda)) .
\end{aligned}
$$

Substituting (68), (71) and (72) into system (62) - (63) we derive system of ordinary differential equations on functions $F(\lambda), H(\lambda), Q(\lambda)$, also containing variables $t, \theta, z$. Knowing that

$$
t=\frac{\left(r^{2} \theta^{2}+z^{2}\right)^{2 / 3}}{\lambda^{2 / 3}}
$$

we can eliminate variable $t$ from derived equations and then split with respect to remaining variables $\theta$ and $z$. Thus we derive the following system:

$$
\begin{aligned}
( \pm 4 Q-3 \lambda) H^{\prime}+H & =0 \\
-8 c_{0} \lambda^{2} F^{\prime}+\left(4 \lambda Q \mp 3 \lambda^{2}\right) Q^{\prime} \pm \lambda Q-2 H^{2}-2 Q^{2} & =0 \\
(\mp 4 Q+3 \lambda) F^{\prime} \pm 2 F Q^{\prime}-c_{0} E \alpha F^{3}+F & =0 .
\end{aligned}
$$

Case 1. If $H^{\prime} \neq 0$, then from $(74)$ we have

$$
Q(\lambda)= \pm \frac{1}{4}\left(3 \lambda-\frac{H}{H^{\prime}}\right)
$$

Remaining equations (75) - (76) take the form

$$
\begin{aligned}
2 \lambda H^{2} H^{\prime \prime}+\left(16 H^{2}+2 \lambda^{2}+64 c_{0} \lambda^{2} F^{\prime}\right) H^{\prime 3}+H^{2} H^{\prime} & =0, \\
F H H^{\prime \prime}+2\left(2-E c_{0} \alpha F^{2}\right) F H^{\prime 2}+2 H F^{\prime} H^{\prime} & =0 .
\end{aligned}
$$

Thus invariant solution of system (62) - (63) has the form

$$
\begin{aligned}
\rho & =t^{-\frac{1}{2}} F(\lambda) \\
u & =\frac{3}{4} \frac{r \theta}{t}-\frac{t^{1 / 2}}{r^{2} \theta^{2}+z^{2}}\left(z+\frac{1}{4} \frac{r \theta}{H^{\prime}(\lambda)}\right) H(\lambda), \\
v & =\frac{3}{4} \frac{z}{t}+\frac{t^{1 / 2}}{r \theta}\left[1-\frac{z}{r^{2} \theta^{2}+z^{2}}\left(z+\frac{1}{4} \frac{r \theta}{H^{\prime}(\lambda)}\right)\right] H(\lambda),
\end{aligned}
$$

where

$$
\lambda=t^{-3 / 2}\left(r^{2} \theta^{2}+z^{2}\right)
$$

and functions $H(\lambda), F(\lambda)$ satisfy system (78) - (79).

Case 2. If $H^{\prime}=0$, then from (74) we derive that $H=0$ and system (74) - (76) takes the form

$$
\begin{aligned}
-8 c_{0} F^{\prime}+(4 Q \mp 3 \lambda) \lambda Q^{\prime} \pm \lambda Q-2 Q^{2} & =0 \\
(\mp 4 Q+3) F^{\prime} \pm 2 F Q^{\prime}-c_{0} E \alpha F^{3}+F & =0 .
\end{aligned}
$$


Thus invariant solution of system (62) - (63) has the form

$$
\begin{aligned}
\rho & =t^{-\frac{1}{2}} F(\lambda), \\
u & = \pm \frac{r \theta t^{1 / 2}}{r^{2} \theta^{2}+z^{2}} Q(\lambda), \\
v & = \pm \frac{z t^{1 / 2}}{r^{2} \theta^{2}+z^{2}} Q(\lambda),
\end{aligned}
$$

where $\lambda$ is given by (83) and functions $F(\lambda)$ and $Q(\lambda)$ satisfy system (84) - (85).

\section{Discussions}

The nonlinear governing gas dynamics equations that are used as a descriptor of a rotating detonation engine were investigated from the group theoretical standpoint. The equations incorporate approximation of the Korobeinkov's chemical reaction model that are used to describe the two-dimensional detonation field on a surface of a two-dimensional cylindrical chamber without thickness. The transformations that leave the equations invariant are found. On the basis of these transformations, the conservation equations were constructed and the invariant solutions were obtained for specific form of the equation of state, for which the equations are nonlinearly self-adjoint. The invariant solutions are given in terms of the functions that satisfy nonlinear ordinary differential equations. The above reduction simplifies the analysis of the original nonlinear system of partial differential equations on a surface of rotating cylinder. Without loss of generality, the parameter $a$ that appears in the transformation were set to the averaged approximated constant speed of sound $c_{0}$ in the media. This parameter can also be used in order to get further reductions to get the approximate forms of the equations, for which the invariant solutions could be obtained in explicit form ([20]; [5]; [4]). Our next goal is to extend the group theoretical modeling to the case, when the equation of state (16) is generalized to the form (5) and so the Eq. (10) will be considered, including the terms of the order of $\varepsilon$ so that we will need the requirement that $T$ is sufficiently large. This will be done in the forthcoming studies and the results will be published elsewhere.

Acknowledgments. This research was supported in part by an appointment to the U.S. Department of Energy's Visiting Faculty Program and by the Government of Russian Federation through Resolution No. 220, Agreement No. 11.G34.31.0042.

\section{References}

[1] Clayton, R.M., 1970: Experimental observations relating to inception of liquid rocket engine popping and resonant combustion to the stagnation dynamics of injection impingement, JPL TR 32-1479.

[2] Davidenko, D.M., Gokalp, I., Kudrryavtsev, A.N., 2008: Numerical study of the continuous detonation wave rocket engine, AIAA Paper, p. 268.

[3] Heiser, W.H., Pratt, D.T., 2002: Thermodynamic cycle analysis of pulse detonation engines, J. Propulsion Power, 18(1), p.68. 
[4] Ibragimov, N.H., Ibragimov, R.N., 2011: Applications of Lie Group Analysis in Geophysical Fluid Dynamics, Series on Complexity, Nonlinearity and Chaos, Vol 2, World Scientific Publishers, ISBN: 978-981-4340-46-5.

[5] Ibragimov, R.N, Jefferson, G., Carminati, J., 2013: Invariant and approximately invariant solutions of non-linear internal gravity waves forming a column of stratified fluid affected by the Earth's rotation, Int. J. Non-Linear Mech., 51, 28-44.

[6] Ibragimov, R.N., 2010: Free boundary effects on stability of two phase planar fluid motion in annulus: Migration of the stable mode. Comm. Nonlinear Sci. Numer. Simulat. 15 (9), 2361-2374.

[7] Ibragimov, R.N. Guang, L., 2015: Splitting phenomenon of a higher-order shallow water theoryassociated with a longitudinal planetary waves, Dyn. Atmos. Oceans Volume 69, $1-11$.

[8] Ibragimov, R.N., 2007: Oscillatory nature and dissipation of the internal waves energy spectrum in the deep ocean. Eur. Phys. J. Appl. Phys., 40, 315-334.

[9] Ibragimov, R.N., 2008: Generation of internal tides by an oscillating background flow along a corrugated slope, Phys. Scr. 78, 065801.

[10] Ibragimov, R.N., 2011: Nonlinear viscous fluid patterns in a thin rotating spherical domain and applications, Phys. Fluids, 23, 123102.

[11] Ibragimov, N.H., Ibragimov, R.N., 2012: Applications of Lie Group Analysis to mathematical modelling in Natural Sciences, Math. Model. Nat. Phenom.7 (2), 52-65.

[12] Ibragimov, N.H., 2007: A new conservation theorem, J. Math. Anal. Appl., 333: 311-328.

[13] Ibragimov, N.H., Ibragimov, R.N., 2012: Rotationally symmetric internal gravity waves, Int. J. Non-Linear Mech. 47, 46-52.

[14] Ibragimov, N. H. 2011: Nonlinear self-adjointness in constructing conservation laws. Archives of $A L G A$ 7/8, 1-99. See also arXiv:1109.1728v1[math-ph], 2011, pp. 1-104

[15] Kailasanath, K., Oran, E.S., Boris, J.P., Young, T.R., 1985: Determination of detonation cell size and the role of transverse waves in two-dimensional detonations, Combust Flame, 61(3), 199-209.

[16] Kinddracki, J., Walanski, P., Gut, Z., 2011: Experimental research on the rotating detonation in gaseous fuels-oxygen mixtures, Shock Waves, 21, 75-84

[17] Korobeinikov V. P., Levin V. A., Markov V. V. and Chernyi G. G, 1972: Propagation of blast waves in a combustible gas, Astronautica Acta 17, 529-53\%.

[18] Massa, L., Lu, F. K., 2011: Role of the Induction Zone on Detonation-Turbulence Linear Interaction, Combustion Theory and Modelling, 15 (3), 347-371.

[19] Nordeen, C. A., Schwer, D., Schauer, F., Hoke, J., Cetegen, B., and Barber, T., 2011: Thermodynamic modeling of a rotating detonation engine, AIAA Paper 2011-0803. 
[20] Ovsyannikov, L.V., 1981: Lectures on the Fundamentals of Gas Dynamics (in Russian), Nauka, Moscow.

[21] Roy, G.D., Frolov, S.M., Borisov, A.A., 2004: Pulse detonation propulsion: challenges, current status, and future perspective, Progress in Energy and Combustion Science, 30, p.545

[22] Shen, I-wu, Adamson, T.C., 1973: Theoretical analysis of a rotating two phase detonation in a rocket Motor, NASA CR 121194

[23] Shcwer, D., Kailasanath, K., 2010: Numerical investigation of rotating detonation engines, 46th AIAA/ASME Joint Propulsion Concerence\%Exhibit.

[24] Taki, S., Fujiwara, T., 1978: Numerical Analysis of Two-Dimensional Nonsteady Detonations, AIAA J. 16(1), 73-77.

[25] Zhdan, S.A., Bykovskii, F.A.„Vedernikov, E.F., 2007: Mathematical modelingof a rotation detonationwave in a hydrogen-oxygen mixture, Combustion, Explosion, and Shock Waves, $43(4)$, p.449. 